\title{
Transcriptomic Analysis of Neurulation and Early Organogenesis in Rat Embryos: An In Vivo and Ex Vivo Comparison
}

\author{
Joshua F. Robinson, ${ }^{*} \dagger$ Aart Verhoef,* and Aldert H. Piersma* ${ }^{*}, 1$ \\ *Laboratory for Health Protection Research, National Institute for Public Health and the Environment (RIVM), 3721 MA Bilthoven, the Netherlands; \\ $\dagger$ Department of Toxicogenomics, Maastricht University, 6229 ER Maastricht, the Netherlands; and $\ddagger$ Institute for Risk Assessment Sciences (IRAS), Utrecht \\ University, 3584 CM Utrecht, the Netherlands
}

${ }^{1}$ To whom correspondence should be addressed. Fax: +31 30274 4446. E-mail: aldert.piersma@rivm.nl.

Received September 20, 2011; accepted November 15, 2011

Cultured embryos mimic the morphological developmental progression of embryos (in vivo) undergoing neurulation and early organogenesis. Using available genomics technologies, comparative molecular-based assessments between cultured embryos and in vivo models may further clarify commonalities and dissimilarities, which contribute to differences between systems. Therefore, in this study, using a transcriptomic approach, we compared cultured whole rat embryos and embryos in vivo at comparable time points in development (gestational day (GD) $10+2-48 \mathrm{~h}$, GD $0=$ copulatory plug) to assess for commonalities and differences in gene expression in relation to morphology. We reveal strong parallels in time-dependent expression of genes in terms of magnitude, directionality, and functionality between whole embryo culture (WEC) and in vivo (rat). Genes changing in expression over time resemble previously hypothesized mechanisms underlying early development in mammalian systems. Furthermore, at the gene and functional level, we identify genes, which differ in expression between models, including genes related to development, oxygen transport, and metabolism. In summary, our results support the use of WEC for toxicological studies aimed at representing in vivo development during this time window at the molecular level. Additionally, we indicate genes, which differ in expression between models, providing possible insights for improvement of culture conditions.

Key Words: development; transcriptomics; whole embryo culture; neurulation; rat; organogenesis; alternative; comparative genomics.

During the past century, whole embryo culture (WEC) has been used for scientific research investigations of early embryogenesis in the fields of developmental biology, pharmacology, and toxicology. WEC provides an advantageous alternative model for experimental research due to the ability to continuously monitor morphological progression throughout the culturing period and the capacity to control parameters such as developmental timing, duration, and exposure (Robkin et al., 1974). For investigations of neurulation and early organogenesis, rat embryos are typically extracted from maternal dams at gestational day (GD) 10 and cultured for 24-48 h (Flick and Klug, 2006). During this time in development, embryos mature exponentially in weight (New, 1978), with protein (Cockroft, 1976; New et al., 1976) and RNA content increasing 10- to 100fold during culture. Using variations of this approach, the WEC model has been used to study normal gastrulation somitogenesis and neural tube formation as well as craniofacial, cardiac, neural crest cell, and eye development (Ellington, 1991; Fleming et al., 1997). Likewise, signaling pathways underlying these processes, such as Wnt (Augustine et al., 1993), Bmp (Mine et al., 2008), Shh (Ribes et al., 2009), and retinoic acid (Ribes et al., 2009) have been investigated using WEC. Furthermore, WEC has been used to study how genetic and environmental factors may alter early embryogenesis. Developmental toxicological studies using model teratogens, such as valproic acid (Tung and Winn, 2011), triazoles (de Jong et al., 2011), phthalates, alcohol (Chen et al., 2011), methanol (Miller and Wells, 2011), and methylmercury (Naruse et al., 1991) indicate the ability of WEC to identify compound effects on embryo morphology and related-genetic mechanisms. Due to the complexity and relevance of developmentally related morphological and molecular changes during this window in early embryogenesis, WEC provides a suitable model to study normal and adverse developmental outcomes due to genetic and environmental influences.

Cultured embryos grow and differentiate in a fashion similar to their in vivo counterpart; however, over time, retardation in development and the inability to sustain growth is apparent (New et al., 1976). Multiple factors may influence WEC sustainability, including serum type, gassing, and supplementation of nutrients. In general, cultured embryos require animalderived serum to develop. Heat-inactivated rat serum remains the gold standard (Ellis-Hutchings and Carney, 2010); however, serum from other sources, including monkey, human, and less animal exhausting and costly alternatives, such as bovine (Flynn, 1987) and serum-free mediums (Moore-Scott et al., 2003) may be used. Gas supplementation is another critical parameter in WEC for maintaining proper $\mathrm{pH}$ levels and 
providing oxygen to the embryo, which requires increased oxygenation with increasing age (New and Cockroft, 1979). To compensate for the lack of maternal transport of specific nutrients, supplementation of vitamins (Cockroft, 1979), amino acids (Coelho and Klein, 1990), glucose (Klug et al., 1985), and iron (Flynn, 1987) may also improve growth during culturing. Further identification and understanding of factors critical for WEC may result in enhanced culturing conditions, therefore, advancing the representation of the WEC model for in vivo comparisons.

The emergence of genomic technologies has provided scientists a tool to assess developmental progression at the molecular level on a global scale. Recent studies have investigated transcriptomic profiles in association with developmental hallmarks, including neurulation and early organogenesis in human (Fang et al., 2010) and mouse (Hartl et al., 2008; Mitiku and Baker, 2007). These studies have identified the global dynamics of gene expression changes across developmental time, including aspects of functionality, directionality, and timing. Furthermore, the generation of these types of studies have resulted in cross comparisons of common genes across in vivo models to explore similarities (and differences) in early development at the molecular level for human and mouse (Fang et al., 2010), mouse and Drosophila (Mitiku and Baker, 2007), and mouse, Xenopus laevis, Gallus gallus, and Danio rerio (Irie and Kuratani, 2011). These relative comparisons suggest conservation of developmental pathways between species during early development and provide examples of the power of genomic-based comparisons to compare the timing of developmental events in embryogenesis across animal models.

Here, in this study, we examined the transcriptome of rat embryos derived from WEC and in vivo during the period of neurulation and early organogenesis. Assessing across six comparable time points in embryonic development (GD $10+$ 2-48 h), we observe the complexity and diversity of gene expression changes, which underlie this critical period in embryogenesis. Additionally, we identify similarities and differences in gene expression in relation to morphology and functionality between experimental models.

\section{EXPERIMENTAL PROCEDURES}

Animal care. As described previously (Robinson et al., 2010; Luijten et al., 2010), animal studies were approved and conducted in accordance with the National Institute for Public Health and the Environment Animal Care facility and federal regulations. Wistar HsdCpd:WU rats (Harlan, the Netherlands) were housed at the National Institute for Public Health and the Environment (RIVM) in a climate-controlled room with a 12-h on/off light cycle (2:00-14:00, dark). After acclimating for at least 2 weeks, virgin female rats were housed with adult male rats for a 3-h mating period (9:00-12:00). Females showing evidence of a copulatory plug (declared GD 0 ) were housed in separate cages. Animals were monitored daily for general health for the extent of the study. Water and food were provided ad libitum.

Rat postimplantation whole embryo culture. Following previously published methods (Anon, 1999; Piersma et al., 2004), on GD 10, euthanization of pregnant dams was performed via intracardiac injection of T61 (Intervet, the Netherlands), and rat embryos were extracted from the uterus with the yolk sac and ectoplacental cone left intact. Embryos with one to five somites were used at the onset of culture and equally distributed based on initial somite number and maternal origin across experimental groups. For gene expression studies, only embryos with two to four somites were used. The preselection of this range of embryos for RNA studies is based on our initial setup of the WEC model as a screening tool (Luijten et al., 2010). Embryos were cultured separately in a serum mixture (S9001, 90\% pregnant bovine serum, $10 \%$ rat serum; Biochrom, Berlin, Germany) diluted with $14 \%$ Hank's solution and supplemented with $1.57 \mathrm{mg} / \mathrm{ml} \mathrm{D-glucose}$ and $75 \mu \mathrm{g} / \mathrm{ml} \mathrm{L}$-methionine (Gibco). The use of bovine serum mixture is suboptimal compared with the use of 100\% rat serum. However, the use of this serum mixture significantly reduces the usage of animals for this experiment. Furthermore, our previous studies indicate that using a variation of this serum mixture ( $90 \%$ bovine and $10 \%$ rat) versus heat inactivated $100 \%$ rat serum, results in only a reduction of approximately one somite after $48 \mathrm{~h}$ in culture (Luijten et al., 2010). Using increasing concentrations of oxygen, gassing occurred five times during culture: on the first day for $30 \mathrm{~s}$ at 9:00 (approximately $-1 \mathrm{~h})$ and 16:00 $(\sim 7 \mathrm{~h})\left(5 \% \mathrm{O}_{2}, 5 \% \mathrm{CO}_{2}\right.$, and $\left.90 \% \mathrm{~N}_{2}\right)$, on the second day for $30 \mathrm{~s}$ at 9:00 $(\sim 23 \mathrm{~h})$ and 16:00 $(\sim 30 \mathrm{~h})\left(20 \% \mathrm{O}_{2}, 5 \% \mathrm{CO}_{2}\right.$, and $\left.75 \% \mathrm{~N}_{2}\right)$, and on the third day for $10 \mathrm{~s}$ at 9:00 $(\sim 47 \mathrm{~h})\left(40 \% \mathrm{O}_{2}, 5 \% \mathrm{CO}_{2}\right.$, and $55 \% \mathrm{~N}_{2}$ ). Cultured in a rotating incubator chamber at constant temperature $\left(37^{\circ} \mathrm{C}\right)$, embryos were monitored regularly using a built-in microscope to assure consistent culturing.

Embryo isolation for gene expression analysis in WEC. Following 2, 4, $6,12,24$, and $48 \mathrm{~h}$ in culture, single embryos were isolated and scored for total somites and development of the neural tube and heart. The yolk sac and ectoplacental cone were completely removed. Isolation and scoring took approximately 3 min per embryo. Embryos collected for each time point were derived from at least three different dams. All embryos used in this study consisted of vehicle control embryos (dimethyl sulfoxide [DMSO], $1 \mu \mathrm{l} / \mathrm{ml}$ ) used in a parallel toxicological investigation (Robinson et al., 2012). Previous studies from our lab indicate that the concentration of DMSO used in this study does not significantly alter embryonic morphology or gene expression (Robinson et al., 2010).

Embryo isolation for gene expression analysis in vivo. Overlapping with WEC experiments temporally, in vivo studies were conducted within the RIVM Animal Care facility. Dams were administered corn oil via oral gavage. Total volumes of vehicle did not exceed $2 \mathrm{ml} / \mathrm{kg}$ body weight. Similar to WEC, rat embryos served as controls for parallel toxicological investigations (Robinson et al., 2012). On GD $10+2,4,6,12,24$, and $48 \mathrm{~h}$, pregnant dams were euthanized with an intracardiac injection of T61 (Intervet), and embryos were extracted from the uterus. All extraembryonic membranes were removed. Embryos were scored for total somites and development of the neural tube.

Embryonic RNA isolation. For both in vivo and WEC studies, after extraction, separate whole embryos were immediately stored in RNAlater RNA stabilization solution (Ambion, Austin, TX) at $4^{\circ} \mathrm{C}$ for 1 week and then $\leq$ $-20^{\circ} \mathrm{C}$ until further processing. To begin RNA isolation, after thawing on ice, each embryo was homogenized by passing through a $26 \mathrm{G}$ needle connected to a 1-ml syringe 10 times. RNA was isolated using the RNeasy Micro Plus RNA Isolation Kit following the manufacturer's protocol for animal tissues (Qiagen). RNA was eluted with $13 \mu \mathrm{l}$ nuclease-free water and stored at $-80^{\circ} \mathrm{C}$. Quantity and quality was measured via the Nanodrop (Nanodrop Technologies Inc., Wilmington, DE) and the 2100 Bioanalyzer (Agilent Technologies, Palo Alto, CA). Total RNA samples with an absorbance value between 1.9 and 2.2 (260/ $280 \mathrm{~nm}$ ) and RNA integrity number $\geq 7$ were used for further analyses. Only embryos with RNA quantity within 2 SDs of each experimental group were chosen for further analyses due to possible loss of embryonic tissue during homogenization or collection.

Microarray hybridization. RNA hybridization and microarray experimentation was performed by ServiceXS B.V. (Leiden, the Netherlands). RNA targets were prepared using $100 \mathrm{ng}$ of purified total RNA as a template for the Affymetrix 3'IVT Express Labeling Kit (901229). Fragmentation reactions 
were performed using $7.5 \mu \mathrm{g}$ complementary RNA (cRNA) for each sample. At a concentration of $0.0375 \mu \mathrm{g} / \mu \mathrm{l}$ fragmented/denatured cRNA, hybridization was conducted using Affymetrix HT RG-230 PM Array Plate (Affymetrix Inc., Santa Clara, CA) according to the manufacturer's instructions. The Affymetrix HWS Kit (901530) was used for hybridization, washing, and staining the chips. In total, 103 arrays were used ( $n=8$ for each of the 12 experimental groups +7 technical replicates).

Microarray analysis-data processing. Quality of microarray images was inspected visually, and raw values were examined for average background scale factors, $3^{\prime} / 5^{\prime}$ ratios of glyceraldehydes 3-phosphate dehydrogenase and $3^{\prime} / 5^{\prime}$ ratios of $\beta$-actin, and NUSE and RLE signal quality metrics. Affymetrix CEL files were normalized using the Robust Multichip Average algorithm using the Brainarray custom CDF version 13 (http://brainarray.mbni.medumich.edu/ Brainarray/Database/CustomCDF) (Dai et al., 2005). Affymetrix internal controls ID were not used in further analyses, leaving a total of 11,521 probe sets for EntrezGeneID mapped genes.

Identification of differentially expressed genes. Normalized data were log transformed and imported into BRB -ArrayTools (Simon et al., 2007). We observed high similarity between technical replicates; therefore, arrays of technical replicates were averaged. Across the 96 arrays (after combining technical replicates), representing 96 separate embryos, we employed three statistical models (ANOVA) to identify genes changing across time independent of model (in both model systems) (MODEL 1) as well as genes changing over time only in the WEC (MODEL 2) and only in vivo (MODEL 3). Additionally, we employed MODEL 1 to identify genes, which differed in expression between model systems.

MODEL $1: \log _{2}\left[\operatorname{Exp}_{n}\right]$ INVIVO_WEC $=B_{\mathrm{TIME}} X_{1}$

$$
+B_{\text {SYSTEM }} X_{2}
$$

MODEL $2: \log _{2}\left[\operatorname{Exp}_{n}\right] \operatorname{INVIVO}=B_{\mathrm{TIME}} X_{1}$

MODEL $3: \log _{2}\left[\operatorname{Exp}_{n}\right] \mathrm{WEC}=B_{\mathrm{TIME}} X_{1}$

An $F$-test was applied for each term, and corresponding $p$ values were calculated. We corrected for multiple testing by determining the false discovery rate (FDR) (Benjamini and Hochberg, 1995). Genes identified to be differentially expressed across time were based on an FDR of $\leq 1 \%$ and an absolute fold change (FC) (Con $\mathrm{Xh} / \mathrm{Con} 2 \mathrm{~h})$ greater than two at any of the six time points $\left(B_{\mathrm{TIME}}\right.$, MODEL $\left.1-3\right)$. Fold change values were determined by calculating the arithmetic mean of each time point group and determining ratios between time point groups and the 2-h time point group.

Genes significantly differentially expressed between models were identified with an FDR of $\leq 1 \%$ and an absolute $\mathrm{FC}$ (in vivo $\mathrm{Xh} / \mathrm{WEC} \mathrm{Xh}$ ) of greater than 1.5 at any of the six time points ( $B_{\text {SYSTEM }}$, MODEL 3$)$.

Hierarchical clustering of genes differentially expressed across time or between model systems was conducted via average linkage and Euclidian distance (TIGR MEV, Dana-Farber Cancer Institute). Principal Components Analysis (PCA) of all genes differentially expressed across time was performed via $\mathrm{R}$ (www.r-project.org).

Functional analysis of genes differentially expressed across time. Genes identified to be significantly changing across time were assessed for enrichment of Gene Ontology (GO) terms (specifically, biological processes) (GenMAPP, Doniger et al., 2003). Significantly, enriched terms were identified to contain at least six genes, $Z$-score $\geq 2$ and $p$ value $\leq 0.05$. GO biological processes were grouped based on GO classification (AMIGO version 1.8) to determine biological process themes. To quantitatively describe changes on a biological process level, we calculated the absolute average FC in respect to the concurrent Con $2 \mathrm{~h}$ of each model system within genes significantly changing over time within each enriched GO biological process (GO-Quant, Yu et al., 2010).

Genes significantly changing across time related to the GO biological process "central nervous system (CNS) development" were examined on the gene level for similar relationships between WEC and in vivo across time (FDR $\leq 1 \%$, absolute $\mathrm{FC} \geq 2$ at any of the six time points). Genes related to CNS development were identified using Database for Annotation, Visualization, and Integrated Discovery (DAVID) (Dennis et al., 2003) for current annotation. Hierarchical clustering of fold ratios ( $\log 2)$ was completed using average linkage and Euclidean distance.

Conservation of CNS developmental expression between WEC, rat, and human. To identify similarities (and dissimilarities) in CNS developmental gene expression between rat WEC, rat, and human, we used gene expression data previously published assessing transcriptomic changes across neurulation/ early organogenesis in human embryos (Fang et al., 2010). Normalized (Robust Multichip Average) data were retrieved from GEO: GSE18887 (NCBI). Average FC values were determined for each gene in respect to S9 ( GD 2021) human embryos. Genes were identified to be significantly altered using Model 4 (ANOVA, FDR $\leq 1 \%$ ).

MODEL $4: \log _{2}\left[\operatorname{Exp}_{n}\right]$ HUMAN $=B_{\mathrm{TIME}} X_{1}$

Comparable staging was determined based on Theiler Stage (EMAGE, Christiansen et al., 2006) and estimated somite number (Moore and Persaud, 1993) during this developmental time period.

Functional analysis of genes differentially expressed Between models. Using a similar approach, we assessed for enrichment of GO biological processes within genes differentially expressed between models. Significantly enriched terms were identified to contain at least four genes, $Z$ score $\geq 2.0$ and $p$ value $\leq 0.05$. GO biological processes were grouped based on GO classification (AMIGO version 1.8) to determine themes. We employed GO-Quant (Yu et al., 2010) to calculate the absolute average FC between WEC and in vivo at each of the six time points within genes differentially expressed between models within each enriched GO biological process. Genes related to enriched GO biological processes: oxygen transport, pattern specification process, amino acid transport, and response to chemical stimulus were identified using DAVID.

Accession numbers. Transcriptomic data of rat embryos undergoing neurulation and early organogenesis are deposited in NCBI GEO under accession number GSE33195.

Morphological scoring. In parallel with RNA expression studies, detailed morphological assessments were conducted on GD $10+48 \mathrm{~h}$ in embryos not used for microarray analysis. In the WEC, embryos with initially $1-5$ somites $(0 \mathrm{~h})(n=12$ embryos derived from eight different dams) were evaluated after $48 \mathrm{~h}$ of culture. Embryos in vivo ( $n=9$, three different mothers) were extracted on GD $10+48 \mathrm{~h}$ as described above and also evaluated. Assessed endpoints included somite number, head diameter (HD), and crown rump length (CRL).

Adopted from previous studies (Brown and Fabro, 1981; Van Maele-Fabry et al., 1990), the total morphological scoring method (Anon, 1999) was used to determine differential changes between WEC and in vivo for developmental morphological hallmarks. Differences in morphology between in vivo and WEC were determined using Student's $t$-tests (two-sided).

\section{RESULTS}

\section{Developmental Progression of Rat Embryos In Vivo and Ex Vivo}

In general, within the first $48 \mathrm{~h}$ of culture, embryos progressed morphologically in a similar fashion as compared with their in vivo counterpart (Fig. 1A). Embryos underwent cranial (q, r, t) and caudal neural tube closure and early organogenesis of the heart, eye, ear, and limb. However, by $48 \mathrm{~h}$, via morphological scoring evaluation, we observed specific regions to be more developed in vivo as compared with WEC, including the 

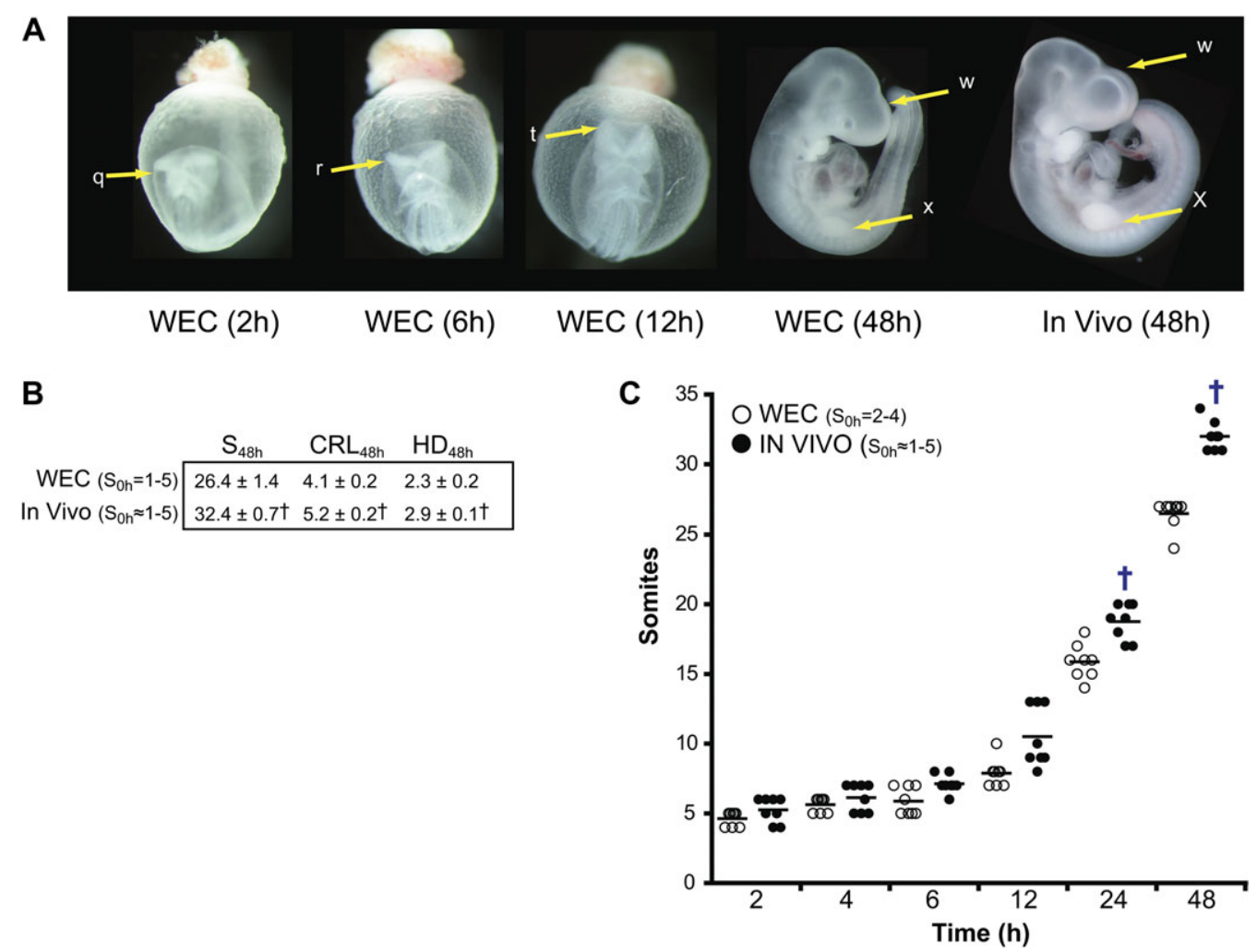

FIG. 1. Morphological development in embryos in WEC and in vivo. Representative examples of embryos in WEC and in vivo across developmental time (A). Arrows point to neural fold development ( $\mathrm{q}, \mathrm{r}, \mathrm{t}$ ) across 2,6 , and $12 \mathrm{~h}$ and embryonic differences at $48 \mathrm{~h}$ between WEC and in vivo (forebrain, w; forelimb, $\mathrm{x}$ ). Mean values and SD for growth parameters of embryos specifically used for morphological assessments at GD $10+48 \mathrm{~h}$, including somites (S), CRL (millimeter), and HD (millimeter) (B). Somitogenesis in single embryos used for microarray analysis (C). Crosses ( $\dagger$ ) signify developmental differences between WEC and in vivo controls ( $\dagger p \leq 0.005, t$-test). Somite $0 \mathrm{~h}\left(S_{0 \mathrm{~h}}\right)$ indicates initial somite stage of embryos used for analysis in WEC and in vivo.

forebrain (w), ear, eye, maxillary, forelimb (x), and hindlimb ( $t$-test, $p \leq 0.05$, values not shown). Morphological differences correlated with larger growth, in terms of somite development (six more somites on average in vivo), CRL, and HD at GD $10+$ $48 \mathrm{~h}$ (Fig. 1B). In embryos used for microarray analysis, across the six time points investigated, a significant increase in somite development in both WEC and in vivo was apparent (Fig. 1C). Significant differences between WEC and in vivo were observed at 24 and $48 \mathrm{~h}(t$-test, $p \leq 0.05)$.

\section{Temporal Changes in Gene Expression During Early Rat Embryogenesis}

Across time, we observed 969 genes to be significantly differentially expressed over the six time points (ANOVA, FDR $\leq 1 \%$, absolute FC $\geq 2$ as compared with the 2-h time point) in WEC and/or in vivo (Fig. 2A). Hierarchical clustering of all 969 genes across time suggested similarities in timedependent directional regulation of gene expression between WEC and in vivo (Fig. 2B). Clusters contained genes that varied in the peak magnitude of change in expression as compared with the 2-h time point, but, in general, we observed genes to increase or decrease in expression across the six time points. On average, the greatest peak changes occurred at $48 \mathrm{~h}$ in both in vivo and WEC (Fig. 2 C). Although subtle $(\leq 0.2$, $\log 2$ scale), greater changes in time-dependent genes were observed at 24 and $48 \mathrm{~h}$ in vivo as compared with the WEC. Using PCA, a time dependence in gene expression changes was observed across the expression profiles of all 96 arrays in WEC and in vivo, moving left to right (Fig. 2D). Along principal component 1 (PC1) which contributed to $77 \%$ of the variability for the expression data of all 969 time-dependent genes, separation between either 48 and $24 \mathrm{~h}$ and earlier time points $(2,4,6$, and $12 \mathrm{~h})$ was clear in both WEC and in vivo. Differences in PC2 that accounted for only $8 \%$ of the variability within the data were apparent between WEC and in vivo at 24 and $48 \mathrm{~h}$.

Significant enrichment of functional groups was observed within the 969 significant time-dependent genes (Fig. 3). Specifically, 112 GO biological processes were identified to be significantly enriched $(p \leq 0.05, Z \leq 2.0$, and number of genes $\geq 6$ ). General themes included, development (general, organ, CNS, embryonic, ear, and circulatory), differentiation (cell and neural), transcription, biological regulation, cell adhesion, lipid/glucose/vitamin metabolism, and transport (e.g., gas). Assessing the absolute average change in expression (as compared with the 2-h time point) within genes associated with each enriched GO biological process, in general, at the functional level, increasing changes in expression were observed 
A

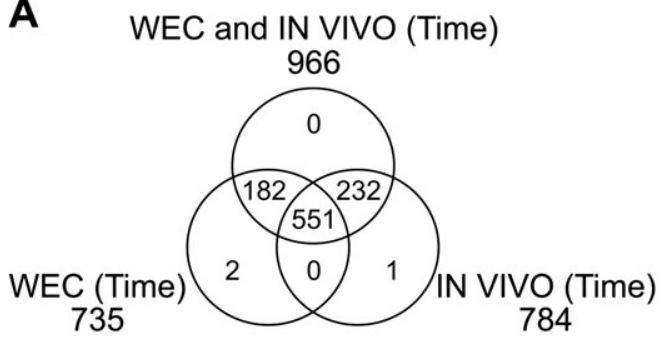

*969 total genes differentially expressed

$(\mathrm{FDR} \leq 0.01, \mathrm{Abs} \mathrm{FC} \geq 2)$
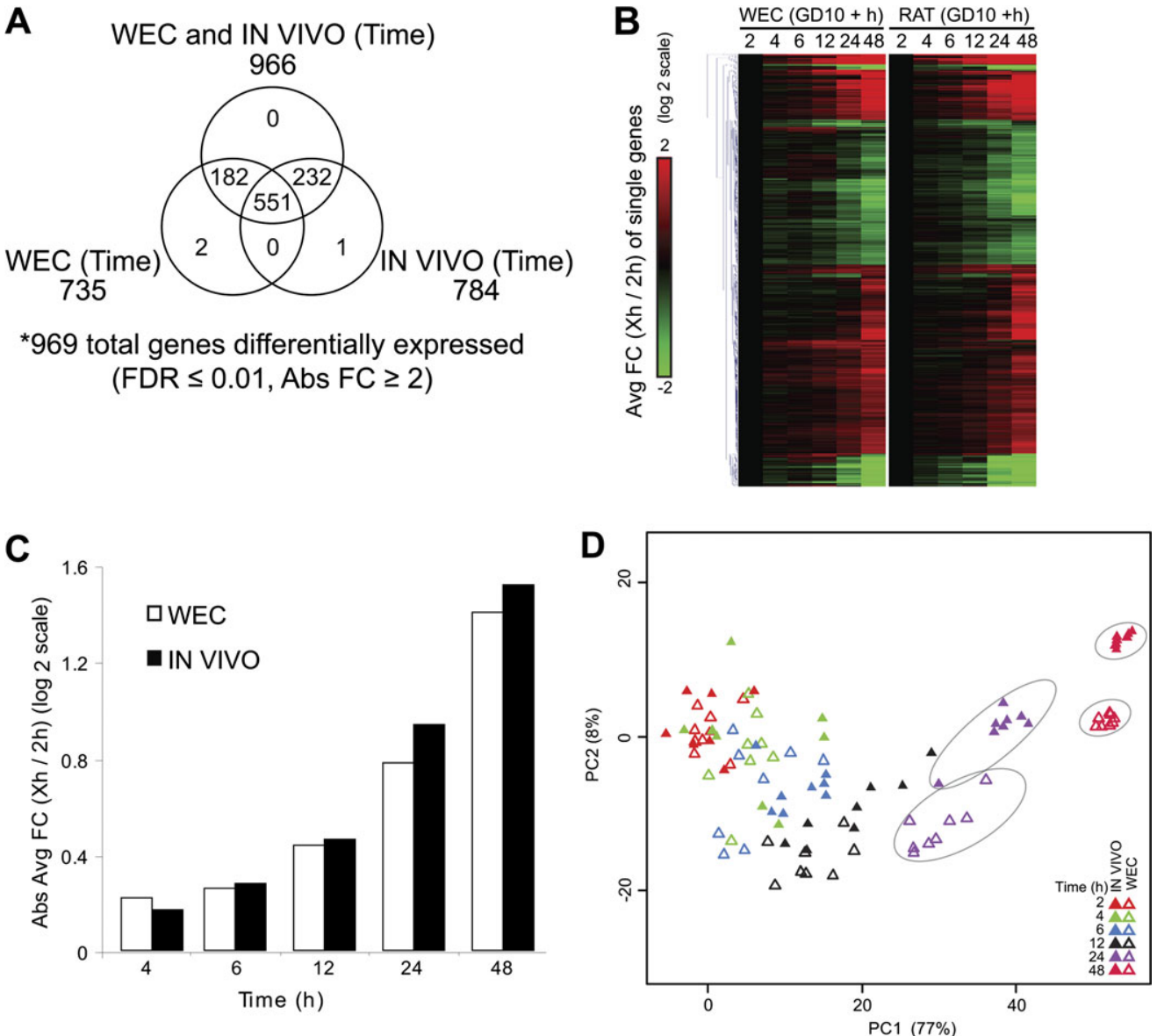

FIG. 2. Time-dependent transcriptomic changes in WEC and in vivo. Number of differentially expressed genes identified to be changing across time in only WEC, in only in vivo, and in both models represented by Venn Diagram (FDR $\leq 0.01$, absolute FC $\geq 2$ ) (A). In total, 969 genes were identified to be significantly changing across time in WEC and/or in vivo model systems. Hierarchical clustering of the average change in gene expression of 969 genes in relation to average 2-h Con in WEC and in vivo (B). Absolute average change in 969 genes identified to be significantly changing over time in WEC and in vivo (C). No error or statistical significance is indicated. PCA of 969 genes significantly changing across time across all 96 arrays (D). Circles emphasize clustering of GD $10+24$ and $48 \mathrm{~h}$ array groups in WEC and in vivo.

in both model systems with increasing time. The largest changes were observed in genes related to oxygen transport, where at 48 $\mathrm{h}$, these six genes had changed in absolute average expression of $31 \times$ and $21 \times$, in comparison with the 2 -h time point, in vivo and WEC, respectively.

\section{Conserved Expression Patterns of Time-Dependent CNS Development Genes in Rat and Human}

As shown in Figure 4A, CNS developmental genes (57 total) significantly changing in expression over time in WEC and/or in vivo were observed to be regulated in the same direction (up or down), and, in many cases, the magnitude was observed to be similar, in terms of FC and temporal regulation. Additionally, directionality of CNS developmental gene expression changes across time as well as the magnitude of changes were similar for multiple genes in comparison with human embryos progressing through a similar developmental period as determined by Theiler Stage and estimated somite number (Fig. 4B). In total, 40/50 genes commonly assessed in rat WEC, rat in vivo, and human showed common significant time-dependent changes (asterisks). Ninety percent of these genes (36/40) were observed to be significantly changing in a similar direction (up/down) across time, including both upregulated (e.g., FABP7, HOXA10) and downregulated genes (e.g., FOXA2, ALDHA2). Exceptions included MAFB, DCLK1, ID4, and VSNL1, which showed common significant changes but differences in directionality of regulation between rat and human.

\section{Model-Dependent Differences in Gene Expression Between Rat Embryos In Vivo and Ex Vivo}

Between WEC and in vivo, we identified 392 genes to be significantly differentially expressed (ANOVA, FDR $\leq 1 \%$, $\mathrm{FC} \geq 1.5$ ) (Fig. 5A). In general, hierarchical clustering of model-dependent genes suggested either genes that were higher or lower in expression between models across all six time points. In terms of magnitude, we observed absolute average differences in expression greater than 0.3 (log 2 scale) between WEC and embryos in vivo at all six time points (2-48 h), with 


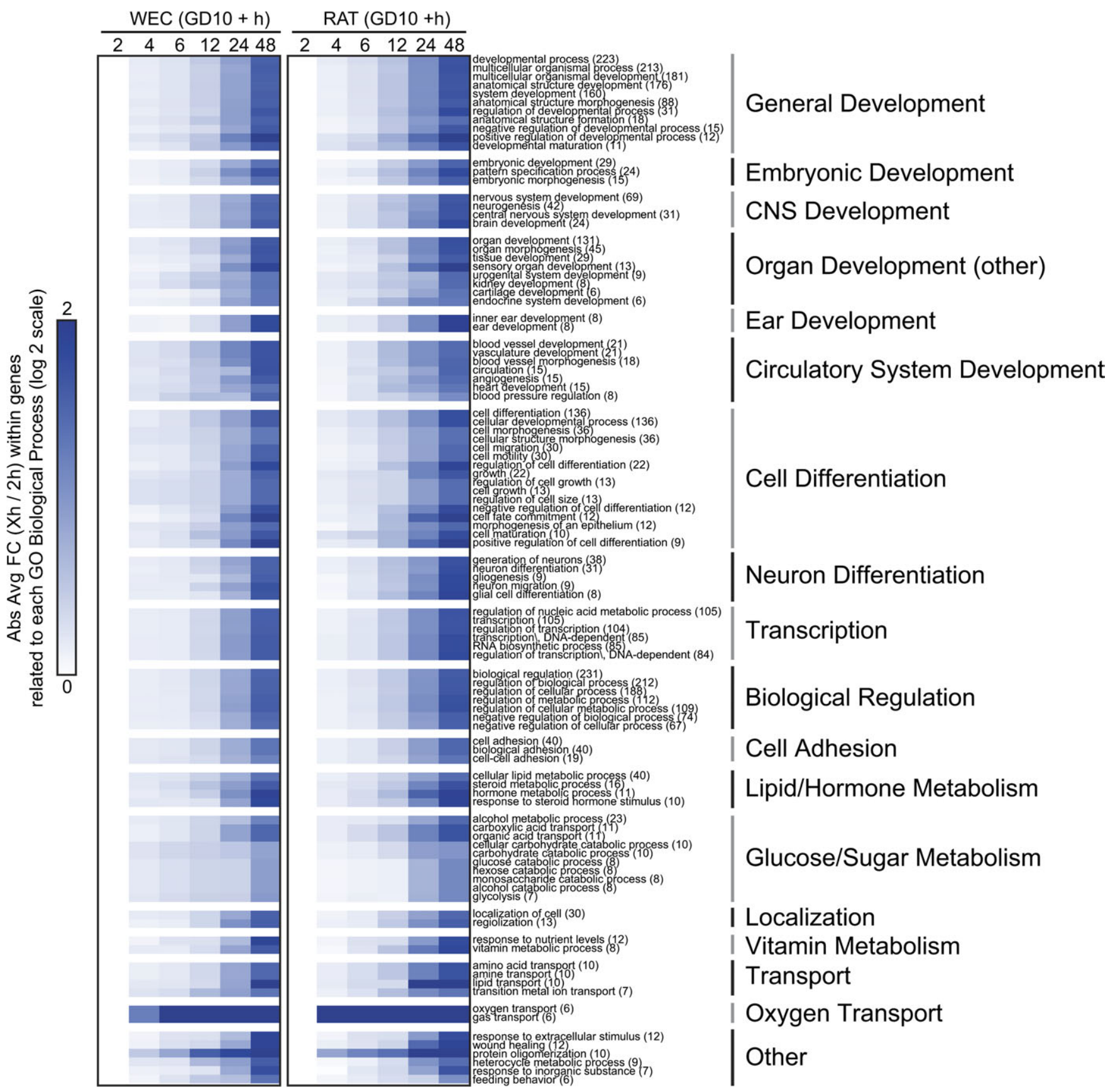

FIG. 3. Quantitative analysis of enriched GO biological process of time-dependent genes. The diagram represents the absolute average degree of change across time within genes related to each enriched GO biological process in WEC and in vivo. In total, 112 GO biological processes were identified to be significantly enriched $(Z \geq 2.0, p \leq 0.05$, number changed $\geq 6$ ) within the 969 genes changing over time (FDR $\leq 0.01$, absolute FC $\geq 2$ ). The number of genes associated with each GO biological process is in parentheses to the right of each GO term. General themes of enriched GO biological processes are to the right of the list of GO terms.

the greatest differences in expression at $24 \mathrm{~h}(0.54 \approx 1.45 \times$ FC) and $48 \mathrm{~h}(0.51)$ (Fig. 5B).

Within model-dependent genes, we observed enrichment of $46 \mathrm{GO}$ biological processes $(p \leq 0.05, Z \geq 2.0$, and number of genes $\geq 4)$. Enrichment analysis suggested differences in genes related to development (general, embryonic, ear, and pattern specification formation), cell differentiation, transcrip- tion, biological regulation, transport (oxygen and amino acid) between WEC and in vivo (Fig. 6). Quantitative analysis on the functional level suggested peak differences in gene expression between genes related to oxygen transport, response to hormone stimulus, and cell differentiation at earlier time points $(\leq 12 \mathrm{~h})$ as compared with genes related to general developmental categories (i.e., system development), 


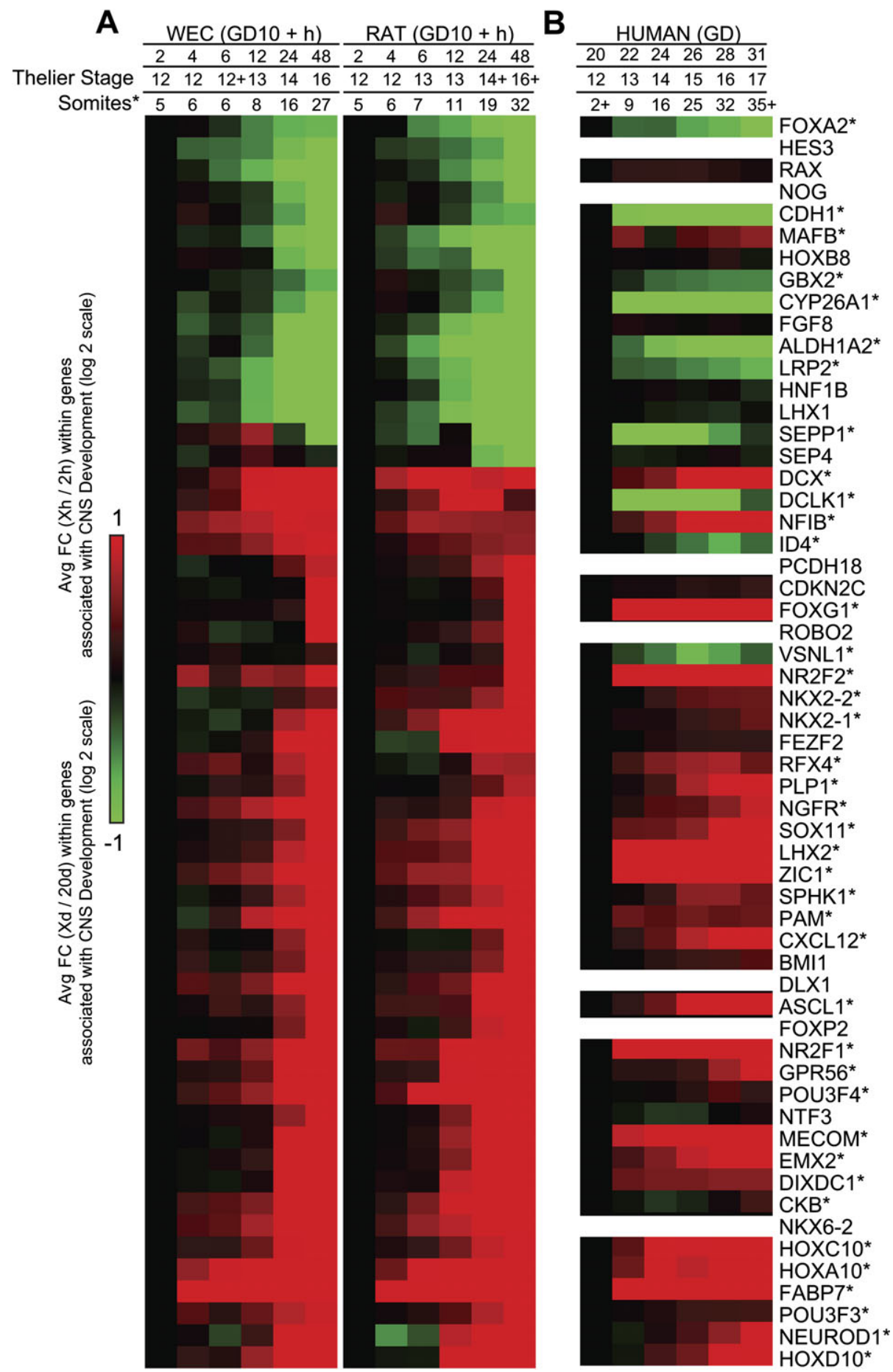

FIG. 4. Time-dependent CNS developmental genes in mammalian systems. Hierarchical clustering of significant CNS developmental genes changing across time in WEC and in vivo (FDR $\leq 0.01$, absolute FC $\geq 2$ ) (A). Comparison of rat-temporal gene expression changes with human embryos undergoing neurulation (Fang et al., 2010) (B). Asterisks $(*)$ near gene symbols represent significant changes in human embryos (FDR $\leq 1 \%$ ).

response to chemical stimulus, cell fate commitment, and heterocycle metabolic process which peaked in differential expression at later time points $(\geq 12 \mathrm{~h})$.

In total, we observed six genes (i.e., GLOB, GLOA, HBB, HBG1, HBE1, and HBE2) related to oxygen transport to be differentially expressed between models (Fig. 7A). All six genes were observed to be expressed higher in vivo as compared with WEC. For example, HBB was observed to be expressed $30 \times$ higher on GD $10+2 \mathrm{~h}$ in vivo as compared with WEC. Differences in oxygen transport genes between 


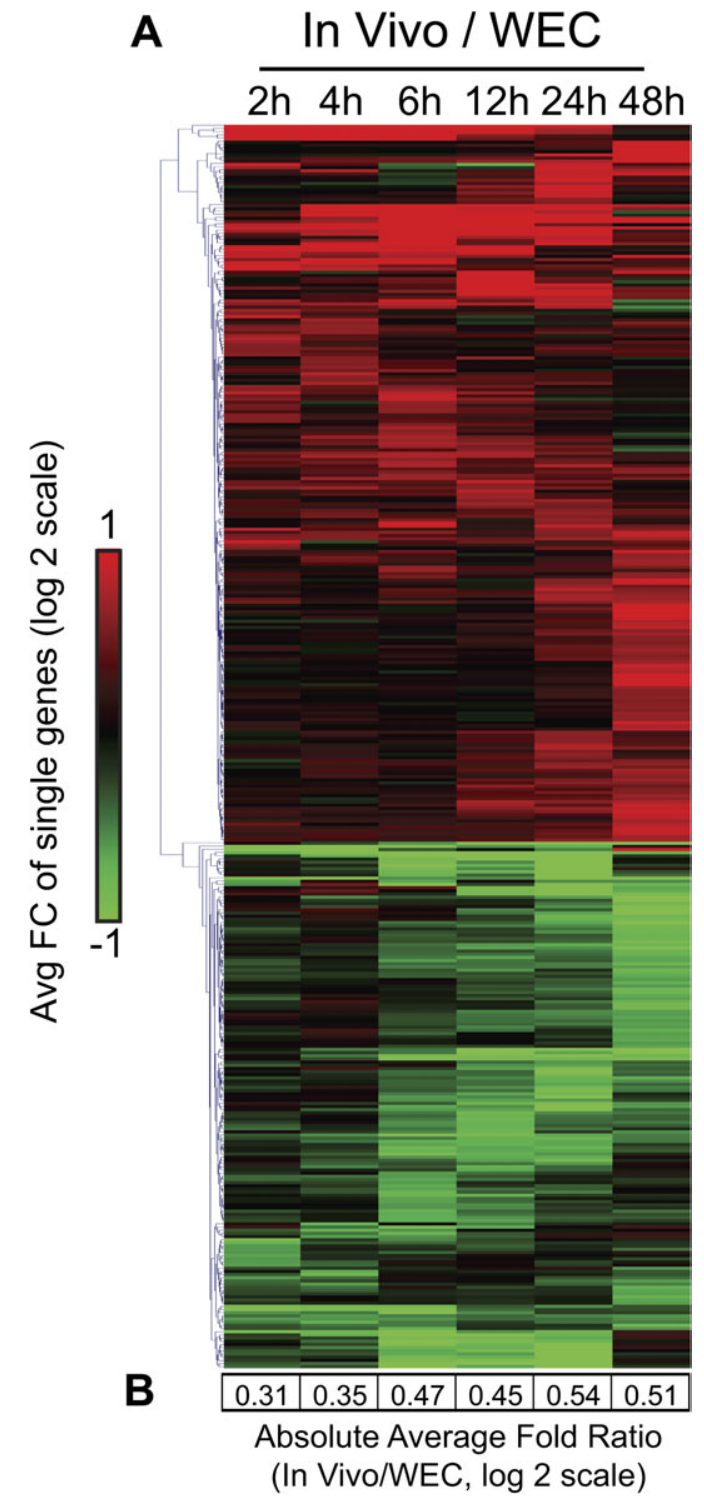

FIG. 5. Model differences in gene expression between WEC and in vivo systems. Hierarchical clustering plot of 392 genes identified to be differentially expressed between models (FDR $\leq 0.01$, absolute FC $\geq 1.5$ ). Shaded intensities represent average difference in expression between in vivo and WEC at each time point (A). The absolute average FC (in vivo/WEC) of all 392 model-dependent genes (B).

models were greatest (except GLOB) at time points $<12 \mathrm{~h}$. Six genes related to pattern specification formation (specifically proximal/distal pattern formation) were significantly expressed higher in vivo as compared with WEC (Fig. 7B). Homeobox paralogs, HOXA10, HOXC10, and HOXD10, were expressed $\sim 2 \times$ higher in vivo as compared with WEC at $12 \mathrm{~h}$. In contrast, all seven genes associated with amino acid transport (SLC7A7, SLC7A1, SLC7A5, SLC7A3, SLC6A15, SLC38A4, and SLC6A6) were observed to be expressed at lower levels in vivo as compared with WEC (Fig. 7C). Oxidoreductase enzymes (10 total) associated with chemical stimuli response were observed to differ in expression between in vivo and WEC (Fig. 7D). In particular, in a time-dependent manner, lower expression of CYP1A1, GPX2, NQO1, CYP2E1, and HMOX1 was observed in vivo versus WEC.

\section{DISCUSSION}

In this study, we presented the dynamics of the transcriptome in single rat embryos derived in vivo and ex vivo progressing over a 2-day window during early rat embryogenesis. Correlated with neural tube formation, somitogenesis, and early organogenesis of the CNS, ear, eye, limbs, and heart (Figs. 1A and C), we observed expression of genes related to multiple developmentally related biological processes to increase in absolute magnitude over time (Fig. 3). Functional gene expression categories included organ development (e.g., CNS, heart, ear) and other processes previously identified to be critical for neurulation (i.e., cell adhesion, transcription, glycolysis, vitamin metabolism) (Copp and Greene, 2010) (Fig. 3). As described in other species, such as human (Fang et al., 2010) and mouse (Mitiku and Baker, 2007), gene expression changes during this developmental period were time-dependent and primarily consisted of either genes increasing or decreasing in expression over time. In particular, we identified multiple transcripts related to different features in CNS development (Fig. 4) as previously described by localization (Christiansen et al., 2006) and functionality studies. For example, well-characterized cranial-region specific (e.g., FOXG1) and caudal-region specific (HOXA10, HOXC10, and HOXD10) CNS transcription factors (Choe et al., 2006; Hebert and Fishell, 2008) increased in expression over time in both models. Furthermore, we demonstrated that changes in expression of specific CNS developmental genes (e.g., FABP7) correlated in a similar manner (directionality, magnitude) in human embryos during a comparable developmental period (Fang et al., 2010) (Fig. 4). Over $80 \%$ of CNS genes identified to be significantly changing over time in rat were observed to be significantly changing over time in human embryos. Our results suggest the ability to detect on a global scale, transcriptional changes reflecting previously hypothesized mechanisms underlying neurulation and early organogenesis in single rat embryos. Additionally, in general, we indicate strong similarities in expression of timedependent genes in rat embryos derived in vivo and ex vivo and, furthermore, suggest conservation of expression of select developmental genes between rat and human embryos during this window in development.

Although time-dependent expression analyses at the functional and gene level, suggested strong similarities between embryos derived in vivo versus ex vivo, we also identified model-dependent differences in expression, which correlated with evident differences in morphological development. At $24 \mathrm{~h}$ (somites) and $48 \mathrm{~h}$ (somites, growth parameter, and specific developmental features), we observed significant differences in morphological development between WEC and embryos in vivo 


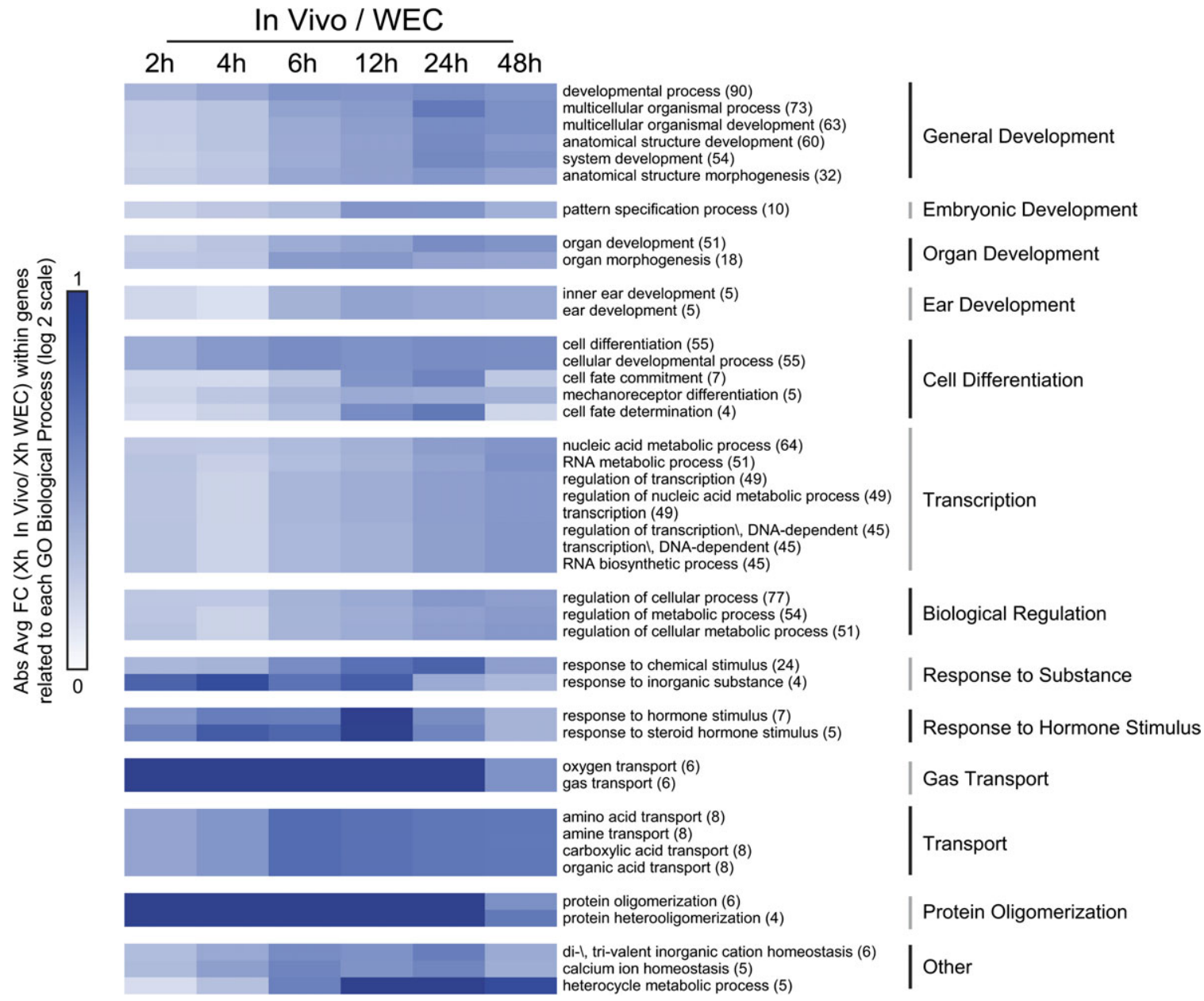

FIG. 6. Quantitative analysis of enriched GO biological process associated with model-dependent gene expression. The diagram represents the absolute average difference in expression between in vivo and WEC within genes related to each enriched GO biological process. In total, $42 \mathrm{GO}$ biological processes were identified to be significantly enriched $(Z \geq 2.0, p \leq 0.05$, number changed $\geq 4$ ) within the 392 genes differentially expressed between in vivo and WEC (FDR $\leq$ 0.01 , absolute $\mathrm{FC} \geq 1.5$ ). The number of genes associated with each GO biological process is in parentheses to the right of each GO term. General themes of enriched GO biological processes are to the right of the list of GO terms.

(Figs. 1A-C). Our observations are supported by previous studies, suggesting eventual growth retardation in embryos using similar culturing procedures. Rat embryos cultured for $\sim 40 \mathrm{~h}$ show a similar decrease in somites $(<20 \%)$ as well as protein content $(<20 \%)$ as compared with in vivo (New and Coppola, 1970; New, 1978). In relation to morphological differences, at the transcriptome level, we observed significant differences in expression of genes related to developmentally related processes as well as other processes of interest (e.g., transport, chemical response) between models (Figs. 5-7). Although differences in expression on average of developmentally related genes were evident between WEC and embryos in vivo at all time points (2-48 h), differences were greatest at 12-48 h (Fig. 6). At the gene level, we provided examples of genes involved in pattern specification, specifically, proximal/distal pattern formation, which included caudal-specific homeobox transcription factors (HOXA9, HOXA10, HOXC10, and HOXD10) and the retinoic acid metabolizing enzyme, CYP26A1, which peaked in expression differences between models $<48 \mathrm{~h}$ (Fig. 7B). Therefore, our results indicate peak differences in expression (or earlier expression) of specific developmental genes between in vivo and WEC, in many cases, precede the largest observed differences in morphology observed at $48 \mathrm{~h}$ (Fig. 1C), suggesting differences in expression (timing or magnitude) may underlie increased development in vivo as compared with WEC.

During the evaluated period of embryogenesis, the yolk sac develops, with fetal blood circulation increasing through surrounding capillaries, which provides a transfer system between the placenta (in vivo) or culture medium (WEC) and the embryo. At the gene level, we observed increased absolute expression of genes related to circulatory development and oxygen transport across developmental time in both models (Fig. 3). Within the six genes related to oxygen transport that significantly increased across time, we observed significantly higher expression of these six genes in vivo in comparison with WEC (Fig. 7C). Differences in expression of these six oxygen 
A

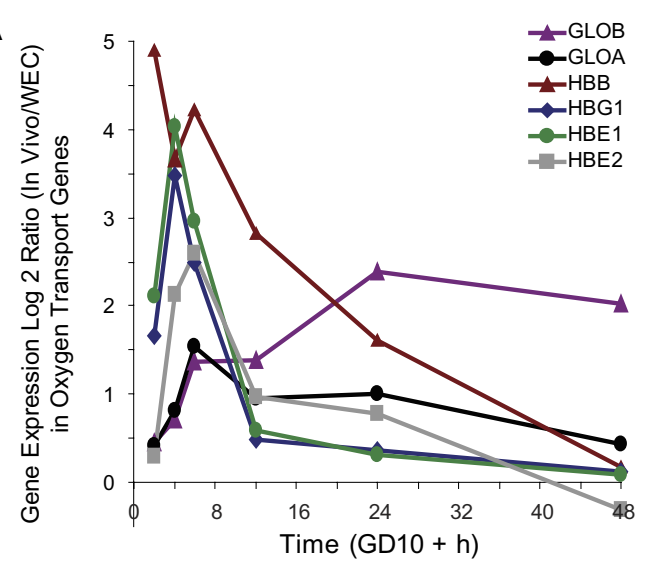

C

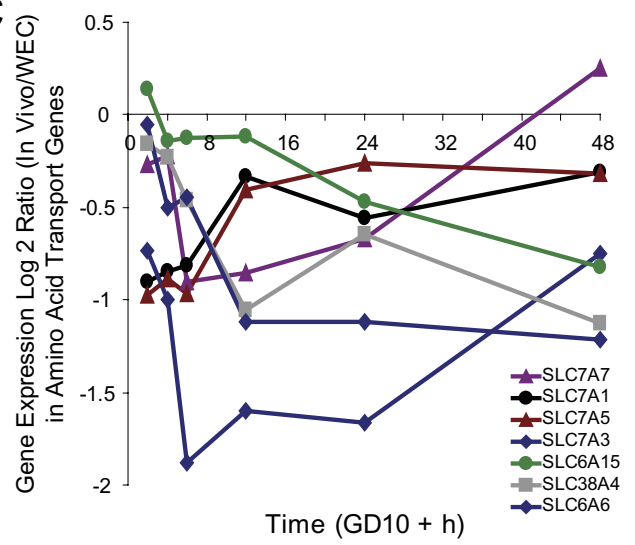

B

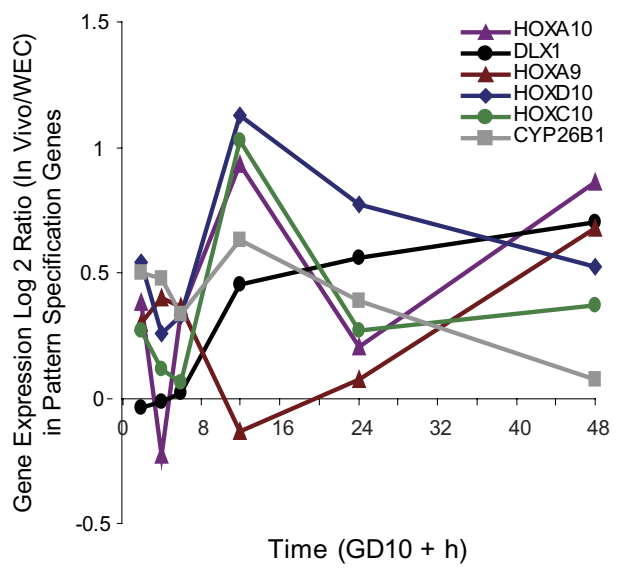

D

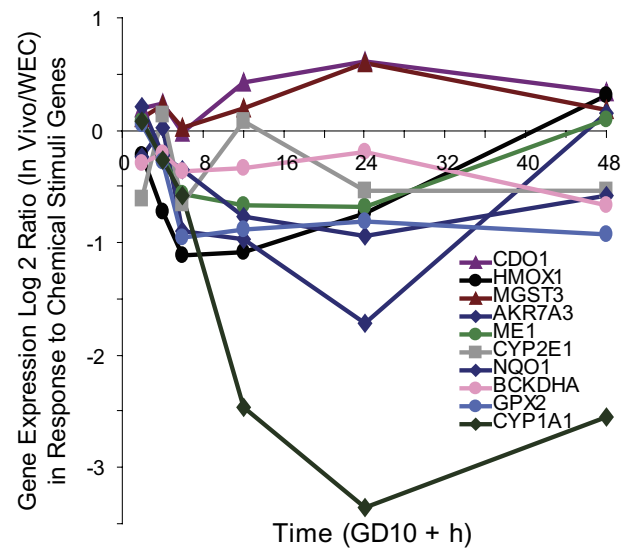

FIG. 7. Model differences in gene expression within enriched GO biological processes between WEC and in vivo. Fold ratios (in vivo/WEC) of genes significantly different between models associated with oxygen transport (A), pattern specification process (B), amino acid transport (C), and response to stimuli (D) across time.

transport genes, which include four hemoglobin (HBB, HBE1, HBE2, and HBG1) and two globin (GLOA and GLOB) family members, correlate with previous studies that have examined the suboptimal nature of hemoglobin production in cultured embryos (Klug et al., 1985). Furthermore, our results suggest early time points in culture to be critical for the expression of oxygen transport genes, with HBB, HBG1, HBE1, HBE2, GLOA all peaking in differences (magnitude) between in vivo and WEC at $<12 \mathrm{~h}$ (Fig. 7B). These observations may suggest relatively anemic conditions in WEC as compared with embryos in vivo during early time periods in culture. Multiple studies have investigated the delicate relationship between embryonic growth and oxygenation levels in culture (Morriss and New, 1979; New and Coppola, 1970). Also, it has been shown that embryos in early stages of culture display transient levels of oxidative stress (Ozolins and Hales, 1997). The oxygenation scheme used in our protocol over the years, with increasing oxygen concentrations with time of culture, was carefully designed for optimal embryo development. Using lower concentrations of oxygen would reduce embryonic progression, whereas higher concentrations of oxygen would result in increased developmental abnormalities. Genes associ- ated with normal oxygen transport identified in this study, in parallel with experimental studies addressing protocol development, may aid in the improvement of embryo culture.

The lack of a functional allantoic placenta, which facilitates maternal-fetal transfer of nutrients, oxygen, and waste products, presents a challenge in producing equivalent embryos in vivo and in vitro. Although not optimal compared with in vivo, the addition of serum promotes gas and nutrient exchange, maintains $\mathrm{pH}$ status, and provides a pool for waste products in culture over time. Within model-dependent genes, we observed significant enrichment of genes related to response to chemical stimuli and amino acid transport (Fig. 6). In particular, we observed higher expression of amino acid transporters (SLC7A7, SLC7A1, SLC7A5, SLC7A3, SLC6A15, SLC38A4, and SLC6A6) in WEC compared with in vivo (Fig. 7C). Higher expression of these genes involved in maintaining the balance of cationic and neutral amino acids in the developing embryo (Hundal and Taylor, 2009) may indicate a lack of needed nutrients and a compensatory mechanism to regulate nutrient levels in WEC. Additionally, we observed higher expression of oxidoreductase enzymes (CYP1A1, HMOX1, NQO1, CYP2E1, and GPX2) in WEC as compared with in vivo (Fig. 7D). Higher 
expression of these enzymes, which are all involved in the detoxification of oxidative products (Dinkova-Kostova and Talalay, 2010) and associated with anemia, may also indicate embryos cultured in vitro to be under greater hypoxic stress as compared with in vivo. These results may suggest differences in sensitivity to chemical compounds between models due to differing conditions. Monitoring the expression of these oxygenand nutrient-sensitive genes, in comparison with normal in vivo conditions, may provide an improved baseline for culturing conditions in vitro.

Improved correlations in the expression of genes between model systems over time may be observed with enhanced culturing conditions, which promote closer similarities in embryonic progression. Two aspects of this study which influenced comparisons between WEC and in vivo included the use of a ratbovine serum mixture and the preselection of embryos with two to four somites for gene expression assessments. Culturing embryos in any animal serum is suboptimal compared with the in vivo condition; however, we estimate that the use of our bovine-rat serum mixture in this study as compared with $100 \%$ rat serum compromised embryonic progression by approximately 1.5 somites after $48 \mathrm{~h}$ of culturing (Luijten et al., 2010), suggesting that the use of rat serum may have improved correlations but to what degree is not addressed in this study. Global molecular investigations comparing cultured embryos in $100 \%$ rat serum versus alternative sera may further define the influence of serum origin/culture media on development in WEC. Additionally, preselection of embryos with two to four somites $\left(S_{0 \mathrm{~h}}\right)$ for WEC-gene expression assessments as opposed to the normal range in vivo ( $S_{0 \mathrm{~h}} \sim 1$ to 5 somites) on GD 10 may also influence differences in gene expression. However, embryos used for gene expression studies $\left(S_{0 \mathrm{~h}}=\right.$ 2-4 somites) as compared with embryos used for morphological assessments in the WEC ( $S_{0 \mathrm{~h}}=1-5$ somites) showed no significant differences in somite number at $48 \mathrm{~h}$ (Figs. 1B and C), suggesting preselection criteria to be of small impact in the WEC. Additional known factors (i.e., gassing schedule, nutrient supplementation) may also reduce retardation of cultured embryos as compared with in vivo, and therefore, may improve correlations at the gene expression level between model systems.

Here, taking a global perspective, we have described the dynamics of the transcriptome in single rat embryos derived in vivo and ex vivo during a critical period in embryonic development, specifically neurulation and early organogenesis. Similar to previous transcriptomic studies conducted in other animal systems, we have demonstrated the complexity of timedependent gene expression changes on a temporal, directional, and functional basis. Furthermore, we have shown the power of transcriptomic approaches to describe molecular similarities on a global scale across animal models and the ability to distinguish differences that are associated with morphological subtleties. As we move forward with the use of alternative models for scientific research (e.g., toxicology), transcriptomic- based assessments that complement traditional morphological assessments may be employed as a measure to determine optimal culturing conditions or "normal developmental status" to verify stability and consistency in the experimental setting. The present study serves as a resource for mechanistic research studies investigating in vivo and in vitro animal model systems during embryogenesis.

\section{SUPPLEMENTARY DATA}

Supplementary data are available online at http://toxsci. oxfordjournals.org/.

\section{FUNDING}

Netherlands Genomics Initiative/Netherlands Organization for Scientific Research (NWO) no: 050-06-510; the Netherlands Toxicogenomics Center.

\section{ACKNOWLEDGMENTS}

We would like to thank Fang et al. (2010) from the Chinese Academy of Sciences and Shanghai Jiao Tong University School of Medicine (SJTU-SM) for use of their dataset in this study.

\section{REFERENCES}

Anon (1999). In INVITTOX Protocol 123. Embryotoxicity Testing in Postimplantation Rat Whole Embryo Culture: Method of Piersma. Available at: http://ecvam-dbalm.jrc.ec.europa.eu/.

Augustine, K., Liu, E. T., and Sadler, T. W. (1993). Antisense attenuation of Wnt-1 and Wnt-3a expression in whole embryo culture reveals roles for these genes in craniofacial, spinal cord, and cardiac morphogenesis. Dev. Genet. 14, 500-520.

Benjamini, Y., and Hochberg, Y. (1995). Controlling the false discovery rate: A practical and powerful approach to multiple testing. J. R. Stat. Soc. B 57, 289-300.

Brown, N. A., and Fabro, S. (1981). Quantitation of rat embryonic development in vitro: A morphological scoring system. Teratology 24, 65-78.

Chen, Y., Ozturk, N. C., Ni, L., Goodlett, C., and Zhou, F. C. (2011). Strain differences in developmental vulnerability to alcohol exposure via embryo culture in mice. Alcohol Clin. Exp. Res. 35, 1293-1304.

Choe, A., Phun, H. Q., Tieu, D. D., Hu, Y. H., and Carpenter, E. M. (2006). Expression patterns of Hox 10 paralogous genes during lumbar spinal cord development. Gene Expr. Patterns 6, 730-737.

Christiansen, J. H., Yang, Y., Venkataraman, S., Richardson, L., Stevenson, P., Burton, N., Baldock, R. A., and Davidson, D. R. (2006). EMAGE: A spatial database of gene expression patterns during mouse embryo development. Nucleic Acids Res. 34, D637-D641.

Cockroft, D. L. (1976). Comparison of in vitro and in vivo development of rat foetuses. Dev. Biol. 48, 163-172.

Cockroft, D. L. (1979). Nutrient requirements of rat embryos undergoing organogenesis in vitro. J. Reprod. Fertil. 57, 505-510.

Coelho, C. N., and Klein, N. W. (1990). Methionine and neural tube closure in cultured rat embryos: Morphological and biochemical analyses. Teratology 42, 437-451. 
Copp, A. J., and Greene, N. D. (2010). Genetics and development of neural tube defects. J. Pathol. 220, 217-230.

Dai, M., Wang, P., Boyd, A. D., Kostov, G., Athey, B., Jones, E. G., Bunney, W. E., Myers, R. M., Speed, T. P., Akil, H., et al. (2005). Evolving gene/transcript definitions significantly alter the interpretation of GeneChip data. Nucleic Acids Res. 33, e175.

de Jong, E., Barenys, M., Hermsen, S. A., Verhoef, A., Ossendorp, B. C., Bessems, J. G., and Piersma, A. H. (2011). Comparison of the mouse Embryonic Stem Cell Test, the rat Whole Embryo Culture and the Zebrafish Embryotoxicity Test as alternative methods for developmental toxicity testing of six 1,2,4-triazoles. Toxicol. Appl. Pharmacol. 253, 103-111.

Dennis, G., Jr, Sherman, B. T., Hosack, D. A., Yang, J., Gao, W., Lane, H. C., and Lempicki, R. A. (2003). DAVID: Database for annotation, visualization, and integrated discovery. Genome Biol. 4, P3.

Dinkova-Kostova, A. T., and Talalay, P. (2010). NAD(P)H:quinone acceptor oxidoreductase 1 (NQO1), a multifunctional antioxidant enzyme and exceptionally versatile cytoprotector. Arch. Biochem. Biophys. 501, 116-123.

Doniger, S. W., Salomonis, N., Dahlquist, K. D., Vranizan, K., Lawlor, S. C., and Conklin, B. R. (2003). MAPPFinder: Using Gene Ontology and GenMAPP to create a global gene-expression profile from microarray data. Genome Biol. 4, R7.

Ellington, S. K. (1991). Use of embryo culture to study normal development and developmental mechanisms. Reprod. Toxicol. 5, 229-235.

Ellis-Hutchings, R. G., and Carney, E. W. (2010). Whole embryo culture: A "New" technique that enabled decades of mechanistic discoveries. Birth Defects Res. B Dev. Reprod. Toxicol. 89, 304-312.

Fang, H., Yang, Y., Li, C., Fu, S., Yang, Z., Jin, G., Wang, K., Zhang, J., and Jin, Y. (2010). Transcriptome analysis of early organogenesis in human embryos. Dev. Cell 19, 174-184.

Fleming, A., Gerrelli, D., Greene, N. D., and Copp, A. J. (1997). Mechanisms of normal and abnormal neurulation: Evidence from embryo culture studies. Int. J. Dev. Biol. 41, 199-212.

Flick, B., and Klug, S. (2006). Whole embryo culture: An important tool in developmental toxicology today. Curr. Pharm. Des. 12, 1467-1488.

Flynn, T. J. (1987). Teratological research using in vitro systems. I. Mammalian whole embryo culture. Environ. Health Perspect. 72, 203-210.

Hartl, D., Irmler, M., Romer, I., Mader, M. T., Mao, L., Zabel, C., de Angelis, M. H., Beckers, J., and Klose, J. (2008). Transcriptome and proteome analysis of early embryonic mouse brain development. Proteomics 8, 1257-1265.

Hebert, J. M., and Fishell, G. (2008). The genetics of early telencephalon patterning: Some assembly required. Nat. Rev. Neurosci. 9, 678-685.

Hundal, H. S., and Taylor, P. M. (2009). Amino acid transceptors: Gate keepers of nutrient exchange and regulators of nutrient signaling. Am. J. Physiol. Endocrinol. Metab. 296, E603-E613.

Irie, N., and Kuratani, S. (2011). Comparative transcriptome analysis reveals vertebrate phylotypic period during organogenesis. Nat. Commun. 2, 248.

Klug, S., Lewandowski, C., and Neubert, D. (1985). Modification and standardization of the culture of early postimplantation embryos for toxicological studies. Arch. Toxicol. 58, 84-88.

Luijten, M., van Beelen, V. A., Verhoef, A., Renkens, M. F., van Herwijnen, M. H., Westerman, A., van Schooten, F. J., et al. (2010). Transcriptomics analysis of retinoic acid embryotoxicity in rat postimplantation whole embryo culture. Reprod. Toxicol. 30, 333-340.

Miller, L., and Wells, P. G. (2011). Altered methanol embryopathies in embryo culture with mutant catalase-deficient mice and transgenic mice expressing human catalase. Toxicol. Appl. Pharmacol. 252, 55-61.

Mine, N., Anderson, R. M., and Klingensmith, J. (2008). BMP antagonism is required in both the node and lateral plate mesoderm for mammalian leftright axis establishment. Development 135, 2425-2434.
Mitiku, N., and Baker, J. C. (2007). Genomic analysis of gastrulation and organogenesis in the mouse. Dev. Cell 13, 897-907.

Moore, K. L., and Persaud, T. V. N. (1993). The Developing Human: Clinically Oriented Embryology, 5th ed. WB Saunders, Philadelphia, PA.

Moore-Scott, B. A., Gordon, J., Blackburn, C. C., Condie, B. G., and Manley, N. R. (2003). New serum-free in vitro culture technique for midgestation mouse embryos. Genesis 35, 164-168.

Morriss, G. M., and New, D. A. (1979). Effect of oxygen concentration on morphogenesis of cranial neural folds and neural crest in cultured rat embryos. J. Embryol. Exp. Morphol. 54, 17-35.

Naruse, I., Matsumoto, N., and Kajiwara, Y. (1991). Toxicokinetics of methylmercury and mercuric chloride in mouse embryos in vitro. Bull. Environ. Contam. Toxicol. 47, 689-695.

New, D. A. (1978). Whole-embryo culture and the study of mammalian embryos during organogenesis. Biol. Rev. Camb. Philos. Soc. 53, 81-122.

New, D. A., and Cockroft, D. L. (1979). A rotating bottle culture method with continuous replacement of the gas phase. Experientia 35, 138-140.

New, D. A., and Coppola, P. T. (1970). Effects of different oxygen concentrations on the development of rat embryos in culture. J. Reprod. Fertil. 21, 109-118.

New, D. A., Coppola, P. T., and Cockroft, D. L. (1976). Comparison of growth in vitro and in vivo of post-implantation rat embryos. J. Embryol. Exp. Morphol. 36, 133-144.

Ozolins, T. R., and Hales, B. F. (1997). Oxidative stress regulates the expression and activity of transcription factor activator protein-1 in rat conceptus. J. Pharmacol. Exp. Ther. 280, 1085-1093.

Piersma, A. H., Genschow, E., Verhoef, A., Spanjersberg, M. Q., Brown, N. A., Brady, M., Burns, A., Clemann, N., Seiler, A., and Spielmann, H. (2004). Validation of the postimplantation rat whole-embryo culture test in the international ECVAM validation study on three in vitro embryotoxicity tests. Altern. Lab. Anim. 32, 275-307.

Ribes, V., Le Roux, I., Rhinn, M., Schuhbaur, B., and Dolle, P. (2009). Early mouse caudal development relies on crosstalk between retinoic acid, Shh and Fgf signalling pathways. Development 136, 665-676.

Robinson, J. F., van Beelen, V. A., Verhoef, A., Renkens, M. F., Luijten, M., van Herwijnen, M. H., Westerman, A., Pennings, J. L., et al. (2010). Embryotoxicant-specific transcriptomic responses in rat postimplantation whole-embryo culture. Toxicol. Sci. 118, 675-685.

Robinson, J. F., Verhoef, A., Pennings, J. L., Pronk, T. E., and Piersma, A. H. (2012). A comparison of gene expression responses in rat whole embryo culture and in vivo: Time-dependent retinoic acid-induced teratogenic response. Advance Access published Jan 18, 2012. doi:10.1093/toxsci/ kfr342.

Robkin, M., Shepard, T. H., and Baum, D. (1974). Autonomic drug effects on the heart rate of early rat embryos. Teratology 9, 35-44.

Simon, R., Lam, A., Li, M. C., Ngan, M., Menenzes, S., and Zhao, Y. (2007). Analysis of gene expression data using BRB-array tools. Cancer Inform. 3, $11-17$.

Tung, E. W., and Winn, L. (2011). Valproic acid increases formation of reactive oxygen species and induces apoptosis in postimplantation embryos: A role for oxidative stress in valproic acid-induced neural tube defects. Mol. Pharmacol. 80, 979-987.

Van Maele-Fabry, G., Delhaise, F., and Picard, J. J. (1990). Morphogenesis and quantification of the development of post-implantation mouse embryos. Toxicol. In Vitro 4, 149-156.

Yu, X., Robinson, J. F., Sidhu, J. S., Hong, S., and Faustman, E. M. (2010). A system-based comparison of gene expression reveals alterations in oxidative stress, disruption of ubiquitin-proteasome system and altered cell cycle regulation after exposure to cadmium and methylmercury in mouse embryonic fibroblast. Toxicol. Sci. 114, 356-377. 